Relato de Caso

CASE REPORT

\title{
Condrocalcinose Articular Familiar ${ }^{(*)}$
}

\section{Familial Articular Chondrocalcinosis}

\author{
Mittermayer Barreto Santiago ${ }^{(1)}$, Liliana Galrão ${ }^{(2)}$, Isabella Lima ${ }^{(2)},{\text { Lucas } \text { Prado }^{(3)} \text { e Melba Moura }}^{(3)}$
}

\section{RESUMO}

Condrocalcinose articular familiar é uma condição clínica caracterizada pela deposição de cristais de pirofosfato de cálcio no líquido sinovial e cartilagens articulares levando à artrite. Descrevemos três membros de uma família com condrocalcinose cujo quadro clínico era caracterizado por artrite intermitente em dois e artrite crônica lembrando artrite reumatóide em um. A avaliação radiológica mostrou calcificações em cartilagens de diversas articulações, particularmente de joelhos. A utilização de colchicina foi suficiente para prevenir as crises de artrite em dois pacientes e o paciente com a forma crônica necessitou uso contínuo de antiinflamatórios não-hormonais. Embora aparentemente rara no Brasil, não afastamos a possibilidade desse dado estar subestimado e sugerimos que seja realizada uma avaliação radiológica articular dos familiares de todo paciente com diagnóstico de condrocalcinose esporádica.

Palavras-chave: condrocalcinose familiar, artrite, pseudo-gota, doença de deposição de pirofosfato de cálcio.

\section{INTRODUÇÃO}

Condrocalcinose é uma situação clínica decorrente da deposição de cristais de pirofosfato de cálcio em cartilagens, particularmente de articulações. O quadro clínico é caracterizado por uma artrite aguda recorrente ou, raramente, crônica que usualmente envolve grandes articulações, principalmente de joelhos. Pode estar associada a uma ampla variedade de enfermidades metabólicas como hiperparatireoidismo, hemocromatose, doença de Wilson, hipotireoidismo, hipofosfatemia, hipomagnesemia. Entretanto, a maior parte dos casos é esporádica, sem doença subjacente ou predisposição familiar.

\section{ABSTRACT}

Familial articular chondrocalcinosis is a disorder characterized by deposition of calcium pyrophosphate dihydrate crystal in synovial fluid and articular cartilage that can cause joint pain and arthritis. We have identified three members of the same family with chondrocalcinosis. The clinical features of the disease were intermittent episodes of arthritis in two patients and polyarthritis resembling rheumatoid arthritis in one member. The radiological evaluation showed calcification in several joints, particularly in cartilages of the knees. Therapy with colchicine was enough to prevent arthritic crisis in two patients and continous NSAID use was necessary to control symptoms in the last one. Familial chondrocalcinosis seems to be rare in Brazil, but we do not exclude the possibility that this figure is underestimated and suggest that in cases of sporadic chondrocalcinosis other members of the family should be fully investigated.

Keywords: familial chondrocalcinosis, arthritis, pseudogout, calcium pyrophosphate dihydrate crystal deposition disease.

A condrocalcinose hereditária ou familiar é uma forma menos comum de condrocalcinose, sendo previamente descrita em países e grupos étnicos distintos, incluindo nações como Inglaterra ${ }^{(1)}$, Tunísia ${ }^{(2)}$, Chile $^{(3)}$, Canadá ${ }^{(4)}$, Suíça $^{(5)}$, Suécia ${ }^{(6)}$, EUA $^{(7,8)}$ e Espanha ${ }^{(9,10,11)}$. Relatamos uma família com essa entidade nosológica no Brasil e descrevemos as características clínicas de sua apresentação.

\section{RELATO DE CASO 1}

Paciente do sexo feminino, 39 anos, branca, com história de artrite, assimétrica intermitente acometendo joelhos, tornozelos, mãos, quadril e articulação esterno-clavicular

\footnotetext{
* Núcleo de Reumatologia da Bahia (NRB), Escola Baiana de Medicina e Saúde Pública (EBMSP). Recebido em 24/06/2003. Aprovado, após revisão, em 04/03/2004.

1. Professor adjunto e doutor da EBMSP e coordenador do NRB.

2. Reumatologista do NRB.

3. Estagiário do NRB.

Endereço para correspondência: Mittermayer Santiago. Núcleo de Reumatologia da Bahia (NRB). Hospital Santa Izabel, Praça Almeida Couto 500, CEP 40000-000 Nazaré, Salvador, BA. E-mail: mitter@svn.com.br
} 
desde os 16 anos de idade. Houve piora do quadro há sete anos. Fazia uso de antiinflamatórios não-hormonais durante os episódios de dor, obtendo alívio sintomático. Ao exame a paciente apresenta-se em bom estado geral, eupnéica, acianótica e anictérica. PA $=120$ x $70 \mathrm{mmHg}$. Exame segmentar sem alterações, exceto pela presença de crepitações à palpação dos joelhos. Exames laboratoriais evidenciaram: hemoglobina $11,6 \mathrm{~g} / \mathrm{dL}$; leucograma: 8.100 células com diferencial normal; plaquetas: 366.000; cálcio sérico 9,1 mg/dL (8,5-10,5); fósforo sérico: 3,5 mg/dL (2,5-4,5); fosfatase alcalina $229 \mathrm{U} / \mathrm{L}$ (50-250); FAN por IFI em HEp-2 negativo, fator reumatóide negativo; HLA-B 27 negativo. RaiosX de mãos, joelhos e quadril evidenciaram a presença de condrocalcinose (Figura 1). Optou-se pela introdução de colchicina $0,5 \mathrm{mg} /$ dia, com melhora da sintomatologia.

\section{RELATO DE CASO 2}

(Irmã da paciente acompanhada no primeiro relato): Sexo feminino, 33 anos, branca, com história de poliartralgia em MCFs, punhos e joelhos, migratória e assimétrica desde os 20 anos de idade, com evolução para edema articular intermitente em joelhos há um ano. Ao exame apresentava derrame articular no joelho esquerdo. O restante do exame fisico era normal. Exames laboratoriais consistindo de hemograma, cálcio, fósforo, fosfatase alcalina, T4, TSH, FAN, HLA-B 27, foram normais ou negativos. Raio $\mathrm{X}$ de joelhos apresentando exuberante calcinose bilateralmente. $\mathrm{O}$ quadro articular ficou controlado após a introdução de colchicina (1 mg/dia).

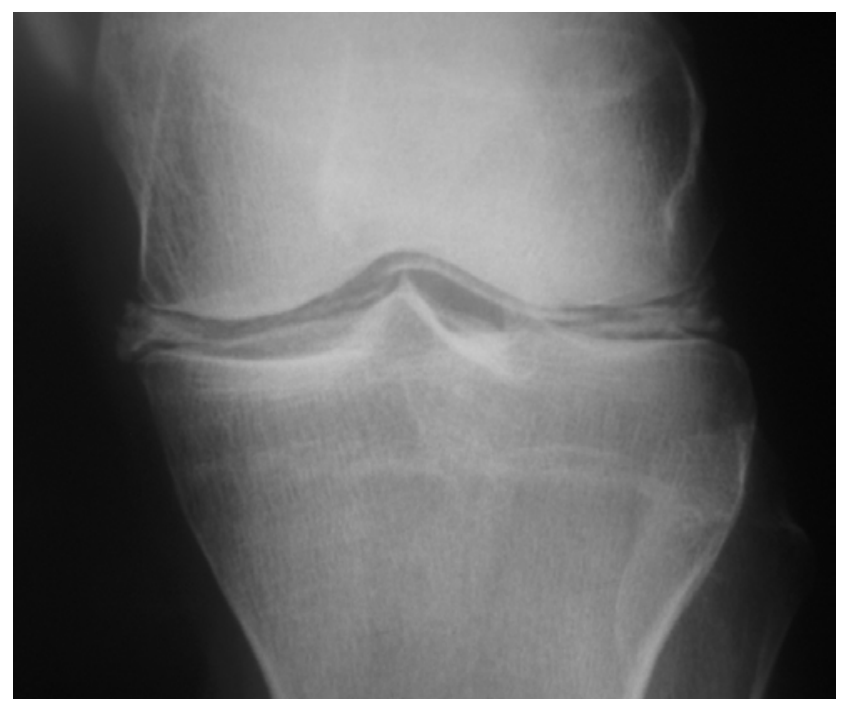

FiguRA 1 - Radiografia de joelho evidenciando extensa calcificação da cartilagem articular, assim como dos meniscos.

\section{RELATO DE CASO 3}

(Tio das pacientes acompanhadas nos dois relatos anteriores): Sexo masculino, 55 anos, branco, com história de poliartrite simétrica em mãos, joelhos e tornozelos desde os 22 anos de idade. Vinha sendo acompanhado em outro centro com diagnóstico de "artrite reumatóide", para a qual fazia uso constante de antiinflamatórios não-hormonais e, eventualmente, corticosteróides, com melhora parcial. Ao exame observava-se apenas aumento de volume e crepitação em joelhos Os exames laboratoriais eram normais e a radiografia dos joelhos também demonstrava calcinose.

\section{DISCUSSÃO}

Condrocalcinose caracteriza-se clinicamente pelo aparecimento de quadro articular inflamatório geralmente intermitente e comumente acomete articulações como joelhos e punhos. Não raramente é observado em pessoas idosas em associação com osteoartrite. Descrevemos três membros de uma mesma família com diagnóstico de condrocalcinose. $\mathrm{Na}$ análise genealógica observamos que alguns outros membros da família apresentavam queixas articulares, sugerindo que essa condição poderia não estar limitada apenas aos pacientes descritos no presente estudo, mas, infelizmente, não foi possível fazer avaliação radiológica dos outros familiares. Essa observação é importante, pois estudo prévio demonstrou que a prevalência de condrocalcinose pode chegar a $26 \%$ em parentes de primeiro grau de pacientes com condrocalcinose familiar ${ }^{(9)}$. Curiosamente, os ancestrais desses pacientes eram de origem portuguesa, como a maioria da população brasileira, e essa condição não foi descrita até o momento em nenhuma família de Portugal.

No Brasil, estudos pioneiros sobre condrocalcinose articular difusa foram desenvolvidos por Nava ${ }^{(12)}$ e Samara ${ }^{(13)}$, embora não se saiba ao certo da descrição da forma familiar dessa doença por esses autores.

Duas pacientes do nosso estudo (irmãs) apresentavam quadro sugestivo de pseudogota, caracterizado por episódios intermitentes de monoartrite, sugerindo a deposição de cristais de pirofosfato de cálcio na articulação, embora o estudo do líquido sinovial para confirmar essa hipótese não pudesse ser realizado. O terceiro membro da família estava sendo acompanhado em outro serviço com diagnóstico de "artrite reumatóide" por apresentar um quadro crônico e poliarticular. O diagnóstico de condrocalcinose foi estabelecido somente após o reconhecimento da existência de condrocalcinose nas duas sobrinhas. Todos os três pacientes tiveram o diagnóstico confirmado de condrocalcinose pelas 
alterações radiológicas características, como ilustrado na Figura 1.

O padrão de herança da condrocalcinose familiar parece ser do tipo autossômico dominante como tem sido sugerido na maioria dos casos e, embora um estudo tenha observado uma maior freqüência do haplotipo A1 B12 DR $3^{(2)}$, parece não haver relação com o HLA ${ }^{(4,14)}$.

Em estudos genéticos de condrocalcinose familiar observou-se inicialmente que a anormalidade genética nessa condição estaria no cromossoma $8^{(7)}$. Porém, outros estudos apontaram para uma anormalidade no braço curto do cromossoma $5^{(15,16)}$ e o provável candidato seria o gene ANKH, homólogo humano do gene que é responsável pela con-

\section{REFERÊNCIAS}

1. Doherty M, Hamilton E, Henderson J, Misra H, Dixey J: Familial chondrocalcinosis due to calcium pyrophosphate dihydrate crystal deposition in English families. Br J Rheumatol 30: 10-5, 1991.

2. Hamza M, Ayed K, Bardi R, et al: HLA-antigens in a Tunisian familial chondrocalcinosis. Dis Markers 8: 109-12, 1990.

3. Fernandez Dapica MP, Gomez-Reino JJ: Familial chondrocalcinosis in the Spanish population. J Rheumatol 13: 631-3, 1986.

4. Gaudreau A, Camerlain M, Pibarot ML, Beauregard G, Lebrun A, Petitclerc C: Familial articular chondrocalcinosis in Quebec. Arthritis Rheum 24: 611-5, 1981.

5. Gerster JC, Schmied PA. Hereditary chondrocalcinosis in a Swiss family Schweiz Med Wochenschr 117: 402-5, 1987.

6. Bjelle A, Nordstrom S, Hagstam A: Hereditary pyrophosphate arthropathy (familial articular chondrocalcinosis) in Sweden. Clin Genet 21: 174-80, 1982.

7. Baldwin CT, Farrer LA, Adair R, Dharmavaram R, Jimenez S, Anderson L: Linkage of early-onset osteoarthritis and chondrocalcinosis to human chromosome 8q. Am J Hum Genet 56: 692-7, 1995.

8. Williams CJ, Pendleton A, Bonavita G, et al: Mutations in the amino terminus of ANKH in two US families with calcium pyrophosphate dihydrate crystal deposition disease. Arthritis Rheum 48: 2627-31, 2003.

9. Balsa A, Martin-Mola E, Gonzalez T, Cruz A, Ojeda S, GijonBanos J: Familial articular chondrocalcinosis in Spain. Ann Rheum Dis 49: $531-5,1990$. dição de anquilose progressiva em camundongo ${ }^{(17)}$. Esse gene codifica a proteína transmembrana que parece regular o transporte de pirofosfato inorgânico ${ }^{(18)}$ e postula-se que a perda da função do ANKH levaria a elevados níveis de pirofosfato inorgânico extracelular e à deposição de cristais de pirofosfato de cálcio ${ }^{(19)}$.

Em conclusão, embora a raridade da observação de condrocalcinose familiar no Brasil possa ter uma explicação genética, a real prevalência da mesma pode estar subestimada e recomenda-se que parentes de primeiro grau de pacientes com condrocalcinose supostamente esporádica sejam submetidos à avaliação radiológica das articulações.

10. Sanmarti R, Panella D, Brancos MA, Anglada A: Hereditary articular chondrocalcinosis in the Osona region (Barcelona): presentation of 3 families Med Clin (Barc) 92: 780-3, 1989.

11. Rodriguez-Valverde V, Zuniga M, Casanueva B, Sanchez S, Merino $\mathrm{J}$ : Hereditary articular chondrocalcinosis. Clinical and genetic features in 13 pedigrees. Am J Med 84: 101-6, 1988.

12. Nava P: Diffuse articular chondrocalcinosis. first cases observed in Brazil. Med Cir Farm 306: 203-10, 1963.

13. Samara AM: Condrocalcinose articular difusa (CCAD). Perspectivas etiopatogenicas. Diffuse articular chondrocalcinosis. Etiopathogenic perspectives. Rev Bras Reumatol 21: 123-6, 1981.

14. Marcos JC, De Benyacar MA, Garcia-Morteo O, et al: Idiopathic familial chondrocalcinosis due to apatite crystal deposition. Am J Med 71: 557-64, 1981.

15. Williams CJ: Familial calcium pyrophosphate dihydrate deposition disease and the ANKH gene. Curr Opin Rheumatol 15: 326-31, 2003.

16. Hughes AE, McGibbon D, Woodward E, Dixey J, Doherty M: Localisation of a gene for chondrocalcinosis to chromosome $5 \mathrm{p}$. Hum Mol Genet 4: 1225-8, 1995.

17. Pendleton A, Johnson MD, Hughes A, et al: Mutations in ANKH cause chondrocalcinosis. Am J Hum Genet 71: 933-40, 2002.

18. Williams CJ: Familial calcium pyrophosphate dihydrate deposition disease and the ANKH gene. Current Opinion in Rheumatology 15: 326-31, 2003.

19. Williams CJ, Zhang Y, Timms A, et al: Autosomal dominant familial calcium pyrophosphate dihydrate deposition disease is caused by mutation in the transmembrane protein ANKH. Am J Hum Genet 71: 985-91, 2002. 